\begin{tabular}{|c|c|c|c|c|c|}
\hline Revista Clio América & ISSN: 1909-941X & Vol. 11 & No. 22 & julio - diciembre de 2017 & 240 - 253 \\
\hline \multicolumn{6}{|c|}{ D0I: http://10.21676/23897848.2444 } \\
\hline
\end{tabular}

\title{
La empresa productiva como espacio de aprendizaje social
}

\author{
The productive company as an area of social learning
}

RESUMEN: El estudio de la empresa ha sido un área de investigación muy importante en las Ciencias Sociales. Por ello, mediante una revision documental interdisciplinaria, se aborda su análisis para la construcción de las bases teóricas de la empresa productiva como centro de operaciones y lugar físico, que demanda de las personas en su condición de empresario, colaborador, a nivel directivo, u operativo interactuar de manera cotidiana y colectiva con otros, aportando conocimiento, experiencia, aprendizaje, sabiduría en el desarrollo conjunto de actividades orientadas a producir los bienes y servicios que la colectividad demanda y necesita para satisfacer sus necesidades. De esta manera, se aborda la teoría de la empresa clásica, con sus antecedentes, el desarrollo de la empresa capitalista, y la Revolución Industrial con la aparición de la máquina, despertando gran interés por la unidades industriales, la construcción de una teoría organizacional donde empresarios y directivos, en razón a la complejidad alcanzada asumen la tarea de darle la dirección correcta, e introducir de manera eficiente las innovaciones producto de la industrialización. Como resultado se obtiene que la empresa es la gran fuente de aprendizaje y vehículo para explotar recursos, coordinar y mantener siempre en aumento una población.

Palabras clave: empresa; trabajo; relación; producción; aprendizaje social.

\section{JEL: L37, N3, L14, L11, D83}

ABSTRACT: The study of the company has been a very important research área in the Social Sciences. Therefore, through an interdisciplinary documentary review, its analysis is addressed to construct the theoretical bases of the productive enterprise as a center of operations and physical location, which demands of the people in their condition of entrepreneur, collaborator, at managerial level, or operative interacting daily and collectively with others, providing knowledge, experience, learning, wisdom in the joint development of activities aimed at producing the goods and services that the community demands and needs to meet their needs. In this way, the classical business theory is approached, with its antecedents, the development of the capitalist enterprise, and the Industrial Revolution with the appearance of the machine, awakening great interest for industrial units, the construction of an organizational theory where Entrepreneurs and executives, due to the complexity achieved assume the task of giving the correct direction, and introduce efficiently the innovations product of industrialization. As a result, we obtain that the company is the great source of learning and vehicle to exploit resources, coordinate and always maintain a growing population.

Keywords: Enterprise; Work; Relationship; Production; Social Learning.

\section{Eliecer Mayorca-Capataz}

Administrador de Empresas, Magister en Administración, candidato al doctorado en Ciencias Sociales mención Gerencia Universidad de Zulia, docente Universidad de Cartagena, Colombia.Email: emayorcac@

$$
\begin{array}{r}
\text { unicartagena.edu.co } \\
\text { ORCID: https://orcid. } \\
\text { org/0000-0003-3647-6388 }
\end{array}
$$

\section{Dolly Yamile Mayorca-Beltrán}

Administradora Industrial, Magister en Ciencias de la Organización, Universidad del Valle, docente de la Escuela de Economía,

Administración y Negocios, Universidad

Pontificia Bolivariana, sede Montería, Colombia. Email: dolly.mayorcab@upb.edu.co ORCID: https://orcid. org/0000-0002-5717-3477

\section{Amalfi Padilla-Castilla}

Trabajadora Social, Doctora en Ciencias de la Educación, postdoctorado en políticas públicas, Universidad de Zulia, docente Facultad de Ciencias Económicas, Universidad de Cartagena, Colombia. Email: apadillac1@ unicartagena.edu.co

ORCID: https://orcid. org/0000-0003-4029-662X

Tipología: Artículo de revisión Fecha de recibido: abril 19 de 2017

Fecha de aceptación: noviembre 07 de 2017 Publicado en línea: noviembre 27 de 2017

Para citar este artículo: Mayorca, C. E., Mayorca, B. D. y

Padilla, C. A. (2017). La empresa productiva como espacio de aprendizaje social. Clío América, 11(22), 240-253. Doi: $10.21676 / 23897848.2444$ 


\section{INTRODUCCIÓN}

La empresa es y seguirá siendo soporte fundamental para los países y sus ciudadanos, porque juega un lugar preponderante en la economía de las naciones. Es así que los historiadores la analizan y narran sus tradiciones, los periodistas la describen, los políticos la debaten y junto con los gobiernos formulan leyes para regular su funcionamiento. Los sindicatos que se crean en su interior hacen peticiones y negocian con ellas, los clientes y consumidores demandan los productos y servicios, a su vez critican o alaban sus prácticas.

Hoy en día la empresa representa el lugar por excelencia donde trabajar la mayoría de las personas y con ello proveer su sustento. Desde los comerciantes de la esquina, que trae el pan y la leche, hasta las pequeñas, medianas, grandes, multinacionales 0 trasnacionales, la empresa siempre está al servicio de nuestras actividades, subsistencia, gustos, salud y bienestar. Es decir, contribuye en cada uno de los aspectos de nuestra vida, su prosperidad está relacionada estrechamente con el cambio y el progreso de la nación (Wren y Greenwood, 1999).

Por otro lado, toda empresa posee una parte fascinante que para muchos consiste en brindar la oportunidad de tender un puente sobre las turbulentas aguas de las clases sociales, al constituirse en el sueño de muchas persona, empezar de la nada y evolucionar hasta alcanzar una posición que le dé estatus, logro, experiencia y riqueza gracias al esfuerzo propio. En segundo lugar, esa fascinación también reside en proporcionar un gran espacio y ambiente de amor y odio con la empresa. Aunque aporta al sustento, se le teme como cualquier otra figura de autoridad y ese carácter dual le ha conferido a la empresa su sabor único a lo largo de la historia (Wren y Greenwood, 1999, p. 9).

Por ello, el ser humano empezó a tallar piedra y pedernales para fabricar armas, a emplear astillas afiladas, utilizar arados de hierros en lugar de maderas, a implementar el uso de la energía hidráuli$\mathrm{ca}$, a poner la presencia de adelantos tecnológicos en el arte de fabricar. Desde siglos atrás se conocía el principio de la energía de vapor; no obstante, el aplicar calor a un líquido y lograr su expansión para transformarlo en un gas para transmitir energía y fuerza, mover objetos y ponerlos al servicio de las necesidades humanas, resulto bastante complejo. Como lo narra la historia del proceso de la invención de la máquina de Thomas Newcomen para bombear agua del fondo de la mina de carbón, debido a numerosos problemas mecánicos.

No obstante, James Watt (citado por Wren y Greeood, 1999), considerado en la época un sabio versado en construir instrumentos, y acostumbrado a trabajar con paciencias, creo la máquina de vapor más eficiente para el momento. Además, los historiadores narran que Wattt, había creado ese prototipo de maquina desde 1765, pero por no disponer del capital necesario para su fabricación, tuvo que postergar por más de una década la producción y comercialización de la máquina de vapor. Hecho que con frecuencia ocurre actualmente en el ámbito de los negocios y demuestra la relación con el otro. Alguien tiene una idea, se une con otra persona que cuenta con el capital y unen esfuerzos para crear una empresa de riesgo al mercado (p.12).

La empresa por consiguiente no es una isla, sino un sector de la vida colectiva que en virtud de unos conocimientos, la ley de especialización del trabajo y con sabiduría, sirve para producir los bienes que la colectividad necesita para su desarrollo (Sánchez, 1990, p. 13). Para ello, la teoría de la empresa como toda área de estudio tiene su génesis y tiende a enmarcarse en las ciencias sociales, con el rigor científico que lo caracteriza, nutriéndose de las distintas disciplinas previas que de ordinario gozan de reputación académica.

De esta manera, cuando se habla en nuestra cultura de empresa, se hace referencia a una entidad legal integrada por el capital, el conocimiento, la experiencia y el trabajo. Con factores de la producción y dedicada a actividades industriales, mercantiles 0 de prestación de servicios, que tienen como objetivo central; mediante la coordinación de recursos humanos, financieros y tecnológicos, la generación de nuevos conocimientos y aprendizajes para obtener beneficios (García y Taboada, 2012, p.10).

Para González (2010), “el vocablo empresa tiene su raíz en el término latín prehendere, que significa 
emprender una cosa que, implica trabajo o presenta dificultades" (p. 9). Además, en la literatura disponible, la empresa es considerada un complejo social dinámico de la economía de un país, que se organiza con la capacidad de generar riqueza y esperanza de empleos. A la vez intuye un gran ambiente y un medio de distribución que influye directa 0 indirectamente en la vida privada y crecimiento de las personas.

La empresa sumida en un entorno dinámico y cambiante, ha sido forzada a evolucionar en el tiempo hasta alcanzar grados de complejidad o simplicidad (Etkin, 2011), que le han permitido adquirir una connotación de pequeña, mediana, grande empresa 0 empresa trasnacional. De esta manera le han otorgado diversos atributos tales como: la empresa viviente (De Geus, 1999), las organizaciones que aprenden (Mayo y Lank 2003), la organización generadora de conocimientos (Nonaka y Takeuchi, 1995), organizaciones abundantes (Ulrich y Ulrich, 2010), fábricas de conocimientos (Fruin, 2000), la empresa intelectual (Stewart, 1997), la empresa consciente (Kofman, 2012), la empresa antropogénica (Jover, 2006) entre otros. Todos estos atributos requieren un análisis de sus dimensiones y alcances en el mundo actual. Por ello, es necesario reflexionar sobre el concepto de empresa para de esta manera situar en ella el intento de formular modelos de intervención.

\section{Antecedentes: la teoría de la empresa clásica}

En la revisión de la literatura, los investigadores y estudiosos del tema señalan como antecedente para la teoría de la empresa a la etapa del surgimiento y desarrollo del sistema capitalista que se inaugura con la Revolución Industrial en Gran Bretaña. Hoy en día, buena parte del impulso a favor de una nueva valoración de la teoría de la empresa, aparece en considerar la Revolución Industrial Británica como el prototipo de industrialización que muchos países han tolerado desde entonces, y otros están centrando grandes esfuerzos en conquistarlo y superarlo para no sufrir en la actualidad (Pollard, 1987, p. 15).

Wren y Greenwood (1999) señalan que cuando se piensa en una revolución, lo que se viene a la mente de las personas es un movimiento súbito y violen- to que trastorna 0 altera los pactos, las alianzas sociales, económicas y políticas. Sin embargo, esta revolución que se produjo en la industria consistió en una serie de cambios ocurridos gradualmente en muchos lugares del mundo a lo largo de más medio siglo. No obstante, el núcleo, la sustancia de lo que se denomina Revolución Industrial fue la maquina a vapor. Los cambios que se produjeron a finales del siglo XVIII en Inglaterra marcaron el inicio de las nuevas tecnologías que fueron decisivas para el desarrollo económico del mundo. (p. 18)

Para Pollard (1987) la experiencia que presenta el interés más inmediato se encuentra entre las numerosas unidades industriales a gran escala que se habían creado a comienzos del siglo XVIII. Las cuales tenían en común, con las empresas que se habían creado posterior a la Revolución Industrial, el haber alcanzado un éxito comercial en un medio competitivo, sin tener que controlar a numerosos trabajadores interactuando y colaborando estrechamente con un equipo muy costoso en un proceso productivo (p.45).

Es el caso de las haciendas agrícolas, estas unidades empresariales que con frecuencia eran muy grandes, con una unidad de producción pequeña formada por una familia campesina, constituyeron la fuente más importante de ingresos individuales, ofreciendo así el marco para la explotación minera y señalar el paso hacia algo más complejo como la manufactura. Esto se produciría al aumentar el tamaño de la empresa, mostrando de esta manera las características de las empresas industriales jóvenes. Proceso que se acelera con la introducción de maquinaria acompañada de una centralización, división y especialización de labores productivas más intensas (García, 1994, p. 26).

Desde este entendimiento, señala Martínez (2005),

suele ser corriente en los textos de la Ciencias Económicas y Administrativas al igual que en ciertas ramas de la Ingeniería como la Industrial, la Ingeniería de Producción y de Alimentos entre otras, concebir la empresa como el lugar físico en donde las personas interactúan de manera individual o colectiva y con el apoyo de maquinaria, equipos y materia prima, se movilizan a realizar tareas orien- 
tadas a la producción de productos o servicios; es decir, la actividad de producción desde el punto de vista etimológico, parece referirse a la operación intencional, en cuya acción vital da por resultado la obtención de un bien o servicio (p.18).

Ahora bien, la producción es algo propio e inseparable de la vida humana, siempre requiere la presencia de un lugar y del ser humano para que con su inteligencia y libertad diseñe procesos abiertos para producir variedad de cosas y de distintas maneras. Para Martínez (2005), esa producción, tal como se observa actualmente, puede que en el plano de su interioridad e individualidad mejore 0 empeore; no obstante, en el plano de lo colectivo y externo puede también crear condiciones que potencian 0 afectan la capacidad de todos. Es decir, la producción es una actividad que hace referencia a unos fines elevados y requiere de experiencia, un mundo previo de conocimientos, una cultura, unos instrumentos y un lenguaje.

Para Negri, (2006), el lenguaje es la única forma de producción de lo humano y de su ambiente; es lo común en el modo de ser de las personas. Por ello, en la actividad de producción se desarrolla mediante la interacción una multitud de actos, donde lo común es el lenguaje que se convierte en la herramienta para transformar o para ponernos en relación con otros, con el contexto y con el mundo. Ese lenguaje supone unas actividades y procesos de producción que sólo son posibles en el seno de una comunidad empresarial mediante un continuado tejido de relaciones humanas y a través de las cosas.

Este hecho da la pista sobre la naturaleza de la producción; se trata de procesos que demandan una red de relaciones, de intercambio y por tanto de un reparto de tareas y funciones en una comunidad (Martínez, 2005). Allí unos se encargan de adquirir la materia prima y los materiales, otros de producir los alimentos, otros en procesar la información; de tal manera que todos ayudan con el fin de vivir una vida lo más humana posible, coordinando de diversos modos la manera de difundir las tareas y organizar los intercambios.

Desde esta perspectiva Douna y Schreuder (2009) señalan que la mayor parte de estos intercambios tiene lugar a través de los mercados, pues ninguna persona es económicamente autosuficiente, en el sentido de producir todos los bienes y servicios que desea consumir; por ello, para obtenerlos debe recurrir a otras personas o instituciones mediante la operación del intercambio. Esta operación de intercambio para Martínez (2005) supone previamente la actividad de producción y presume un beneficio para una comunidad que mantiene 0 incrementa los intercambios, orientado a adquirir los bienes y servicios necesarios con mira a facilitar el establecimiento de una vida humana sostenible. Esta institución es la empresa que realiza un tipo de actividad productiva 0 de servicio, el cual regulan la diferencia entre el flujo de entrada y salida de dinero que provocan la compra de bienes y servicios.

\section{La empresa}

La empresa es el centro o sitio de operaciones de las personas; es el escenario físico en el cual actúan, se relacionan, ponen en práctica conocimientos, adquieren experiencias, nuevos aprendizajes, perfeccionan sus habilidades y enriquecen su personalidad.

Desde la perspectiva clásica, Ortueta (1972), afirma que “las empresas son el primer factor dinámico de la economía de un país y constituyen a la vez un medio de distribución que influye directamente en la vida privada de sus habitantes. Esta influencia justifica la transformación actual, más o menos rápida a que tienden las naciones (p.79)."

Es decir, la empresa es un medio y una forma de organización especial que tiene como propósito ligar las cosas a los hombres, mediante el establecimiento de ciertas relaciones; también de utilización, conservación y potenciación de las fuerzas productivas de la energía humana.

En esta comprensión es significativo el aporte de Drucker (1978), para quien las empresas son órganos estructurados que existen para satisfacer necesidades específicas de la sociedad, generar esperanza de trabajo para la humanidad, obtener unos bienes y servicios que crean valor a los accionistas. La empresa como órgano especial estructurado existe para producir un desempeño económico, pero este desempeño de ningún modo es la única tarea que 
debe cumplir en la sociedad. Además, tiene un carácter prioritario, porque de todas las restantes tareas sociales (educación, atención a la salud, defensa y desarrollo del conocimiento) dependen del excedente de recursos económicos (ganancias y otros ahorros) que sólo pueden obtenerse mediante un desempeño de hacer las cosas lo mejor posible.

Tradicionalmente la empresa se entiende como un acuerdo productivo, con una función claramente definida que es la producción de bienes y servicios, para ser vendidos en el mercado y consumidos por otras personas distintas de quienes participan directamente en su producción. Generalmente dispone de una función de producción que admite desde el autoabastecimiento, la especialización, el intercambio que conduce al aumento de las oportunidades, de elegir para quienes consumen y en definitiva el bienestar de toda la comunidad (Salas, 2007, 141).

De igual manera señala Drucker (1978), para la empresa cumplir su tarea de producir bienes y servicios económicos, debe influir sobre la gente, las comunidades y la sociedad. Para ello, la empresa tiene como recurso autentico y potencial el hombre, quien con sabiduría, conocimiento y experiencia actúa confiriendo productividad a los recursos y realiza su desempeño mediante el trabajo. Por lo tanto, obtener un trabajo productivo es una tarea esencial. Ahora bien, ese trabajo debe ser apropiado para el ser humano y permitirle realizar su potencial como medida de rendimiento de la empresa, siendo una persona que tenga cualidades específicas, capacidades y limitaciones, así como un modo diferenciado de hacer las cosas.

\section{La empresa en la perspectiva institucional}

En el análisis de la empresa productiva, también se encuentra la perspectiva institucional que según Pantoja y Arévalo (2006) es una "corriente institucional, que tiene como fundamento la construcción de una teoría organizacional donde el hombre representado en la figura del empresario, unido a la toma de decisiones como problema de prioridad traza el camino y la dirección que considera correcta de lo que debe mover al país, como elemento clave para la incluir la empresa moderna en el desarrollo de la economía (p.71)."
Según North (1994) la empresa como institución al formar parte de "la estructura de incentivos de una sociedad, y de las instituciones políticas, económicas" (p.288) y sociales, permite que exista una estructura transaccional dentro de la economía, la cual busca integrarse a una dinámica que viene dada por los procesos de aprendizaje, los precios y en consecuencia constituye para Friedman y Friedman (1998), "los determinantes básicos del desempeño para el cambio económico (p.38)."

Al respecto North (1994) considera que en la vida el tiempo tiene un valor ilimitado para el desempeño, y en relación con el cambio, ya sea económico 0 social, se constituye en la dimensión vital para que las personas; bien en calidad de empresario, directivo, u operario, con la capacidad de aprendizaje modelen la evolución de las instituciones. La posición que agrupó originariamente a los fundadores de esta escuela tiene como supuesto que la conducta del ser humano es condicionada por el medio social en el que se desenvuelve de manera cotidiana y a la vez determina que ciertos comportamientos se puedan categorizar como hábitos o costumbre. $Y$ en respuesta a ciertos estímulos del entorno, los individuos actúan de manera rutinaria estableciendo el surgimiento de las instituciones.

Lo anterior, según Veblen (1965), es un llamado de atención para que la economía se aparte de los conceptos tradicionales de equilibrio estático y en su efecto fortalezca la capacidad de aprendizaje de los integrantes de las instituciones, para que se apropien de todo lo relacionado con la evolución del cambio y se adapten. En ese sentido, definió una institución como la entidad que establecen sus propias reglas, normas y reglamentos, y emiten información para guiar el comportamiento de las personas.

Este comportamiento, para Claude (2005), se constituye en hábitos o procesos de pensamientos rutinarios, por ser comunes persisten en la generalidad de las personas. Además son compartidos en las instituciones o empresas por un gran número de personas que con el transcurrir del tiempo se convierten en hábitos, costumbres y más tarde en la manera de influir para guiar y direccionar el comportamiento, que para Martínez (2005) siempre es asociado por las 
personas a la elaboración de las cosas y en la estructura social, modelar las preferencias y los valores de los individuos (p.161)." Pero este enfoque histórico recibe la influencia de la comunidad académica hacia fines de 1920 y comienzos de 1930 que aunado a los posteriores apogeos de la tecnología social, ha contribuido a promover nuevos enfoques analíticos para abordar las instituciones sociales y modificar de manera significativa las obras de los fundadores.

De esta manera, los economistas pertenecientes a la corriente principal (Coase, 1937; Alchian, 1965; Demsentz, 1967; Williamson, 1991, y North, 1994) se percataron de que las instituciones tornan un factor importante para dar explicación a las problemáticas económicas y sociales. Además las determinantes de dichas instituciones son susceptibles de ser analizados por las herramientas económicas ortodoxas, permitiendo una apertura a las instituciones sociales y potenciar desarrollar nuevas capacidades para entender y explicar la realidad, así como el surgimiento del enfoque denominado nuevo institucionalismo.

Este nuevo enfoque considera la naturaleza de las instituciones y tiene como sustento la acción individual de las personas, que inmersa en un contexto social, es parte integrante de la naturaleza humana y es la razón por lo cual Pantoja y Arévalo (2006), “consideran que la nueva economía institucional tenga como referente y punto de encuentro, los fundamentos económicos de los clásicos" (p. 72). En este contexto los individuos tienen una naturaleza interactiva y las instituciones como agentes contribuyen a coordinar sus acciones a través de la dirección; siendo relevante en la interacción las transacciones formales e informales de los individuos que minimizan los costos.

En este sentido, es pertinente el aporte de Commons (1931) quien de manera general concibe la institución, como el proceso de gestión colectiva, donde el cumplimiento de los objetivos tiene como propósito controlar, liberar y ampliar la acción individual. El vínculo entre lo individual y lo colectivo, según Salas (2007), constituye el núcleo principal del interés por la economía. Esto emana de las relaciones de interdependencia como resultado de haber optado por la división del trabajo y la consiguiente especialización de las personas, y satisfacer sus necesidades, tienen la posibilidad de intercambiar con otros especialistas los resultados del trabajo individual.

De esta manera, la colaboración entre especialistas es imprescindible para las tareas a realizar en cada puesto de trabajo, se conviertan irse en bienes y servicios apropiados a las necesidades que desean cubrir (Salas, 2007). En consecuencia, la especialización, la colaboración y el intercambio son tres elementos inseparables de la organización económica. Exigen reglas que permiten crear un orden colectivo a partir de las actuaciones individuales y en consecuencia crean una necesidad de organización para el conjunto de sistema, pero esta organización debe dar respuesta a la necesidad de coordinación.

Esta necesidad de coordinación llevo a Hall (1986) a sistematizar unos elementos conceptuales de "tipo histórico-institucional, pero con el alcance y la capacidad de poder explicar con fundamento en los procesos históricos las variaciones en las políticas de los diversos países. Define las instituciones como reglas formales, procedimientos de aceptación y prácticas operativas normalizadas que estructuran las relaciones entre los individuos y las diversas entidades del sistema de gobierno y de la economía (Hall, 1986, p.19)."

Esta definición de institución representa no sólo la constitución y las prácticas políticas formales, sino también se hace extensiva a redes organizativas menos formales y es la razón para que la empresa empiece a ser concebida como un instrumento común de vinculo, de trabajo, de amistad, que articula la vida de las personas en calidad de cliente, accionista, empresario, directivo o colaborador, consumidor para crecer en comunión.

Como resultado de analizar el enfoque de Hall (1986) se puede afirmar que el institucionalismo representado en la empresa como organización especial y en instituciones del gobierno, es fruto de un encuentro de la historia con la teoría de las organizaciones, ambas buscan abrir las puertas de la vida mediante el acercamiento de las personas. Así mismo, todas las instituciones son interdependientes dentro de una sociedad, puesto que cada una está organizada y estructurada alrededor de un conjunto de normas, valores y pautas de comportamientos; en donde 
la acción colectiva se enmarca desde el propósito de orden natural hasta intereses planificados y programados.

Ahora bien, "un punto en común tanto en la empresa como en las instituciones, es la acción individual, que siempre se encuentra adherida a los comportamientos de acciones colectivas" (Natoli, 2004, p.111), las cuales ayudan a poner magia de sabiduría, aceptación, amistad, experiencia y de conocimiento en la vida de las personas. Transformando así la vida de los demás mediante el desempeño individual en el contacto dentro de una colectividad, compartiendo unas reglas de funcionamiento que establecen los modos de actuar en la producción y los resultados de un bien o un servicio. Este análisis proporciona según Commons (1931) "la correlación entre la ciencia económica, la ciencia jurídica y la ética; además constituye el prerrequisito para una teoría de la economía institucional (p.193)."

Por otro lado, la investigación económica y administrativa ha prodigado que en el mercado se encuentre la principal solución a los problemas que plantea la organización y en razón a ello ha centrado esfuerzo en estudiar el funcionamiento y la forma concreta de abordar la solución a los problemas de coordinación y motivación (Salas, 2007, p.18). Pero el mercado tiene fallas tanto en los aspectos de coordinación como de motivación, porque no entiende sobre criterios de equidad en la distribución de la riqueza creada. De tal manera que los equilibrios a la que confluye la actuación descentralizada no siempre satisface las condiciones de la eficiencia colectiva. Situación que llevó muy pronto a la ciencia económica a justificar la actuación con la intervención del Estado como solución para alinear los intereses individuales y colectivos (Alcaide, 2015, p.182), tardando bastante tiempo en reconocer y valorar los aportes de la empresa a la solución de los fallos de coordinación y otras ineficiencias del mercado, quedando relegada a una mera representación de tecnologías de producción.

Ahora bien, ignorar la empresa en el estudio de la producción y el intercambio no tendría mayor consecuencia si dentro de ella se reprodujeran los mecanismos de funcionamiento propios del mercado. Pero la observación y la constatación de que la empresa funciona y con reglas internas claramente diferenciadas de las que imperan en el mercado llevo a Coase (1937, p. 29) a centrar esfuerzos investigativos hasta publicar La naturaleza de la empresa y marcar en ella la inflexión en el estudio independiente de la empresa dentro de análisis económico y advertir que las personas incurren en costos para descubrir los precios y cerrar los acuerdos sobre los términos de intercambios, denominando costos de mercado.

Coase (1937), considera el momento desde que el mercado funciona y nos acerca al cliente se incurren en costos, ya sea por generar, procesar y utilizar información. Lo relevante ahora es si esos costos son mayores o menores a los que se incurre en el mercado y por medio de este mecanismo ver la posibilidad de poder explicar que "la razón de beneficio principal para establecer una empresa, tiene como fundamento la existencia de un costo en el mecanismo de precios. Y la empresa pone toda su luz para ser iluminada en un sistema de relaciones que se desarrolla cuando la administración y dirección de los recursos depende del empresario (Coase, 1937, p.34)."

Por otro lado, Williamson (1991) plantea el dilema entre mercados y jerarquía como alternativa de solución a los problemas de organización que surgen de la especialización, la colaboración y el intercambio. La jerarquía es la estructura organizativa que prolonga la autoridad del empresario en la dirección y se ubica en el vértice superior de la misma. Sostuvo que es posible entender las fuerzas y los límites del mercado, así como los métodos internos de la organización, sólo si se analiza cada uno en relación con el otro; la relación se centra en hacer económico el costo de transacción y no en la tecnología ni en los costos de producción. De esta manera, sienta las bases sobre la teoría de los costos de transacción que fundamenta en la toma de las decisiones de elección institucional entre empresa y mercados.

Por medio de la teoría de los costes de transacción, Williamson (1991) propone estudiar la organización económica tomando como unidad primitiva la transacción, el cual define como la transferencia entre unidades tecnológicamente separables. La elección 
entre mercado y empresa se plantea en términos de decidir si las unidades entre las que se producen la transferencia se mantiene en empresas separadas 0 alternativamente se integran las dos en una misma empresa. En este sentido Salas (2007) propone que para explicar la elección institucional es necesario conocer los atributos de las propias transacciones y características de las personas que toman las decisiones.

Recopilando las ideas dispersas, la exposición previa contiene referencia a la empresa y al empresario. La empresa es una rica institución social que aparece descrita como un lugar para la comunidad (Sánchez, 2002, p.216) que con magia establece relaciones internas fuertes que le dan cierta estabilidad en el tiempo, dentro del cual transcurren determinadas transacciones. Mientras que la influencia del mercado es dirigir las relaciones entre empresas. En cambio el empresario aparece como la persona física o grupos de personas que en una organización social con división de trabajo realizan funciones de coordinación, supervisión y contacto para mejorar la labor organizativa que colectivamente la sociedad le encarga al sistema de mercado.

Para Salas (2007) al incorporar la perspectiva jurídica se hace más evidente la separación entre empresa y empresario. Para el derecho, la empresa es por encima de toda una entidad jurídica que posee atributo claves de personalidad legal tales como: el poder legal de contratar, de poseer bienes en propiedad, participar en los contratos con el resto de propietarios, sustituyendo a la persona física del empresario. Por otro lado, la estandarización de las normas jurídicas define un marco conocido por todos dentro del cual produce la relación de la empresa con terceros, coordina los comportamientos del mercado, el capital, la publicidad, economiza en costos de transacción y da seguridad a las transacciones.

\section{La empresa industrial moderna}

Las empresas están abiertas a la acción fecunda de un ser superior que mediante el conocimiento, colaboración y cercanía con las personas abre las puertas iluminando la mente humana con ideas, pensamientos, experiencias para renovar siempre a favor de la vida, y lo institucionalizan para el bien la sociedad. Es el caso de las innovaciones surgidas en los medios modernos de transportes y comunicaciones (ferrocarril, telégrafo, buque de vapor, el cable) en el periodo comprendido de los años setenta al ochenta del siglo XIX que, al ser acogido y relacionado en las empresas con la eficiencia para la obtención de resultados, éstas recibieron la recompensas de poder innovar, mejorar y lo más importante transformar la visión de la empresa, con una concepción industrial moderna y una nueva forma de capitalismo.

La conceptualización de la empresa industrial moderna es atribuida al historiador y sociólogo Chandler (1987). Él en sus estudios argumenta que este tipo de empresa obedece a la necesidad que tienen las organizaciones de gestionar de manera eficiente las innovaciones surgidas como producto de la modernización e industrialización de los medios de trasportes y comunicación. Explorando la expansión de los servicios debido al mayor flujo de bienes, de pasajeros y mensajes que los nuevos sistemas de trasportes y comunicación posibilitan para el contacto y movilización de las personas.

Es innegable que para lograr una gestión eficiente de las innovaciones (exploración, adquisición, producción y usos de los medios de trasportes y comunicación) la empresa industrial moderna exige un cambio de adecuación, ampliación y expansión de la planta física del sistema de producción y administrativo. Requiriendo, según Chandler (1996), "las inversiones masivas para la construcción y expansión de sistemas de operaciones de mayor dimensión y complejidad (p.9)." Esto permite a la empresa crear espacios de nuevos departamentos o unidades para asignar un lugar a las personas e integrar una verdadera comunidad y llevar a cabo las distintas operaciones tanto de producción como administración y de esta manera ayudar a crear la historia de la empresa.

También habría que decir que la expansión y creación de nuevas unidades en la empresa industrial moderna no fue sólo a nivel interno, en forma vertical, y en las áreas funcionales, a nivel horizontal; sino también a nivel geográfico, apoyado en las ideas relativas de los principios de la Administración industrial de Fayol (1930) y de la eficiencia de Emerson (1913). 
Además, la incorporación de nuevas unidades en la estructura de la empresa industrial moderna para hacerle frente a las necesidades del entorno, fomenta la flexibilidad y cierto grado de iniciativa en todos los niveles de la escala jerárquica, hecho que lleva a vincular personas para que se pongan al frente de las nuevas unidades. Presentando de esta manera "la separación entre propiedad y dirección, que pasa a ser gestionada por equipos de directivos contratados para coordinar y controlar el trabajo" (Chandler, 1996, p.9).

La hospitalidad en la empresa industrial moderna sobre las innovaciones en el transporte y las comunicaciones, además de la complejidad en el sistema administrativo y de producción, también generó cambio en la forma de concebir al propietario, que además de empresario, pasó a ser inversionistas y tiene que relacionarse para construir un centro de confianza que le permita apropiarse de la información. "Disponer de la experiencia y el tiempo necesario para tomar decisiones adecuadas y necesarias" (Chandler, 1996, p.9).

Las recompensas recibidas por empresarios y directivos de la empresa industrial moderna, por la acogida de las nuevas formas de transportes y comunicación, fueron la consolidación de la estructura organizacional, con los niveles de toma de decisiones representado en nivel directivo, el nivel de mandos medios y el nivel operativo. También la potenciación del sistema de producción que por su gran volumen y eficiencia pasó a un sistema de producción en masa, alcanzando las actividades industriales gran dinamismo para dar respuesta a las demandas del mercado y facilitar el paso a una economía industrial moderna a partir de la mitad del siglo XX (Chandler, 1987, p.32).

Otro aspecto a considerar en el marco de las recompensas de la empresa industrial moderna fue que a medida que incorporaba nuevas unidades a su estructura básica, fuese horizontal, vertical 0 geográfica, el resultado generaba más divisiones y por consiguiente más fácil diversificación productos y servicios (García, 1994, p.33), pero también mayor el tramo de control para la coordinación de las actividades. Tal vez este aspecto es el de más grande soporte para la separación de la propiedad por parte de los empresarios y compartirla con los directivos contratados, alterando de esta manera la estructura básica con la incorporación de los niveles directivos.

Por ello, afirma Zuluaga (2009) "que la empresa industrial moderna fue producto de innovaciones que se tradujeron en desarrollo tecnológico, potenciaron los sistemas de producción haciéndolos más eficientes y la acogida de una clase ejecutivos que la moldearon y le dieron forma (p. 457)." De esta manera, el camino recorrido en este trabajo permite afirmar a manera de síntesis y parafraseando a Drucker (1978) que ninguna empresa existe por sí misma y como un fin por derecho propio, sino que cada una constituye un órgano de la sociedad, que existe para beneficio social. En ese sentido, la empresa se explica por los beneficios que aporta a la sociedad: suministran bienes y servicios, espacios para que las personas en calidad de directivos, operarios, desarrollen tareas que le permitan aplicar conocimientos, obtener experiencias, relaciones, aprendizaje y derivar recursos financieros para las personas y accionistas; por todas estas consideraciones es que forman parte de la comunidad.

\section{La empresa desde la perspectiva sistémica}

La complejidad de la empresa industrial moderna constituye el gran referente para introducirnos en la perspectiva de sistema; ya que, según Chandler (1996), la empresa es considerada "una agrupación de unidades operativas y administrativas (p.28)," en cuyo escenario se construyen y tejen muchas relaciones entre las personas, unidades y departamentos que la integran. Es decir, la empresa se constituye en un centro complejo de contacto, de vínculo a nivel interno entre directivos y colaboradores y a nivel externo con los clientes y grupos de interés.

Dentro de estos planteamientos es de considerar que las personas en su condición de cliente, operarios, directivos, empresarios, inversionistas y en general colaboradores, son el centro y motor que dinamizan la empresa. Esa dinámica se manifiesta en la interacción de las personas ya sea de manera grupal o individual en el desarrollo de las tareas requeridas para elaborar productos o la prestación de servicios y son las razones para considerar la empresa como un sistema social (Bertoglio, 2004, p. 35). 
Por otro lado, la concepción de la empresa como sistema social refuerza la idea de que no se puede hacer operativo la división del trabajo, ni diferenciar las tareas. Tampoco hacer eficiente el sistema de producción como principio organizativo, tal como lo expone Katz y Kahn (2008, p. 94), pues se necesita el apoyo de otras personas con mayor conocimiento, experiencias, sabiduría y aprendizaje. Así como de la tecnologías y de otras instituciones, del entorno en el cual opera la empresa. De tal manera que la interacción permita hacer de la empresa una red de relaciones, de vínculos y donde el contacto permanente genere acercamiento y confianza en las personas unir esfuerzo al trabajo grupal, el aprendizaje y hacer más productivo y eficiente pero sobre todo más humano el sistema de la empresa.

No obstante, habría que agregar, el grado de complejidad alcanzado por la empresa industrial moderna, unido a la complejidad de las condiciones sociales de nuestra época y la velocidad del desarrollo tecnológico, motivaron un nuevo enfoque de la empresa a ser concebida como sistema abierto de relaciones que interactúa con el contexto en el cual está inmersa y del que recibe, insumos, experiencia, conocimientos y al que provee productos de acuerdo a las nuevas exigencias o servicios para el bienestar de la comunidad (Banchik, Rainero y Escudero, 2007, p. 205).

Esta motivación del análisis de la empresa es enriquecida con la perspectiva espiritual en ese sentido Juan Pablo II en su encíclica Centesimus Annus (1991), la define como la unidad productiva en la que se involucran personas en calidad de directivos, operarios, con experiencias y conocimientos, inversionistas que aportan capitales o institución con vocación de servicio y permanencia en el tiempo, orientada a satisfacer necesidades mediante la producción de bienes y servicios, logrando con la actividad beneficios económicos, así como el papel del empresario y del proceso directivo.

La empresa representa el lugar físico por excelencia donde trabajan la mayoría de las personas. Es el lugar de esperanza en donde las personas mediante el trabajo aprenden, aplican los conocimientos, obtienen nuevos, aportan experiencia, dan refuerzos (Jover, 2006, p.218) y derivan unos recursos financieros y con ello proveer el sustento de todos. Es decir, la empresa contribuye a cada uno de los aspectos de nuestra vida y su prosperidad está relacionada estrechamente con el progreso y desarrollo de un país (Melé, 1991, p.3). Además, la visión económica del hombre igual que la sociológica y la política es importante, pero insuficiente, y resalta que la esfera de la cultura provee una mejor comprensión del hombre que la ciencia económica y administrativa; no obstante la interrelación de todas ellas es vital.

Por medio de la encíclica Juan Pablo II sugiere que para una buena organización de la empresa y de los sistemas económicos y administrativos, ha de partirse de una recta concepción de la persona humana, de su relación y dependencia de Dios, de reconocer la existencia de un ser superior que ilumina al hombre en sus pensamientos. De ahí, la necesidad de ver al hombre en su globalidad, considerar la naturaleza y la finalidad de la empresa.

Por consiguiente, las decisiones empresariales para atender la realidad globalmente, no pueden limitarse a buscar únicamente la eficacia económica o la eficiencia en la empresa, teniendo en cuenta el proceso productivo y a la persona como sujeto de normas y reglamento, ventas o consumo, sino que han de tener en cuenta el respeto por la dignidad humana y todos los aspectos relevantes para enfrentar los problema y generar bienestar en la comunidad (Melé, 1991, p. 4). Para Juan Pablo II el desarrollo humano tiene una necesaria dimensión económica, pero no deriva ni se reduce únicamente a lo económico. Él sugiere que para descubrir el verdadero sentido del desarrollo humano es preciso contemplar al hombre de manera integral y considerar su interioridad en el cual se encuentra su naturaleza específica. Y a partir de allí la empresa al igual que las demás instituciones sociales ha de procurar en la medida de las circunstancias lo permita el desarrollo del personal incluido en su grupo de interés.

La empresa al igual que las demás instituciones en el desarrollo de su actividad debe abrir las puertas para que todos sus integrantes puedan, con auténtico espíritu de servicio, de cooperación, trabajar y desarrollar lazos de amistad y evitar generar egoísmos o cualquier otro malestar (Tockman, 2006, p.158). En ese sentido, Melé (1991) reconoce el papel esencialmente positivo de la empresa y de 
otros elementos inseparables de la misma, como el mercado, la propiedad privada y la libre creatividad, las relaciones y el aprendizaje. Para Juan Pablo II la empresa es uno de los elementos básicos para la configuración de una sociedad distinta, tanto del sistema económico basado en el predominio del capital, como los medios de producción respecto a la libre subjetividad del trabajo del hombre. La alternativa a estos sistemas es una sociedad que esté basada en la participación, el trabajo libre y en la empresa.

La empresa es un elemento configurador de la sociedad. A partir de los años 80 con los procesos de apertura económica y la globalización, le han otorgado una gran prioridad que es considerada fuente de todos los progresos, la prosperidad y proveedora de empleo, impulsora de cambios, nuevos aprendizajes y conocimientos (Laval y Dardot, 2013, p. 292). Lo más inmediato a esa prioridad fue la privatización, cuyo argumento es que la empresa tiene mayor eficiencia que la actividad individual, pero la eficiencia es sólo una razón económica, tampoco una justificación global; puesto que hay actividades eficientes económicamente, pero a la vez muy inmorales.

Por lo tanto, la existencia de la empresa, antes de considerar sus actividades, exige también unos fundamentos éticos. Condición que no es difícil para sus dirigentes, si se enfoca desde el sentido común, ya que cualquier persona sensata podría dar muchas razones convincentes de cómo las empresas, si actúan correctamente con buenos principios y cuidado sobre la naturaleza aportan prosperidad: bienes, empleos a la sociedad, buenos ejemplos, buenas prácticas, cambios, valores, cooperación, aspectos no sólo de carácter económico.

Por ello, la orientación de la actividad económica hacia el bien común y al servicio del hombre, hace comprender el verdadero alcance de la propiedad privada y de las libertades económicas (Melé, 1991, p. 6). En definitiva, la empresa es una organización humana que adornada y valorada en todas sus cualidades, es una estimuladora vital para que las personas pongan su magia hacia el cambio y el progreso mediante: eficiencia económica, prosperidad, relaciones y bienestar a favor del bien común. Las personas por medio de ella tienen la oportunidad de establecer nexos de solidaridad y amistad para el crecimiento humano; es decir, la empresa es medio de desarrollo personal, de aprendizaje y expresión de sociabilidad humana.

Hoy inmersa en la sociedad del conocimiento y aprendizaje, la empresa es comprendida en un proceso de organización y construcción relacional abierto, donde el lenguaje que emerge de acciones individuales y grupales la hacen ocupar un papel fundamental para asignarle diferentes denominaciones: empresas que aprenden, empresas creadoras de conocimientos, empresas inteligentes, fábricas de conocimientos (Vidal, 2006, p.38). Es en el conocimiento que la empresa centra sus esfuerzos y esperanza para cimentarse como empresa competitiva. Por ello, lo permite, lo incorpora, lo acoge para renovar y transmitir estabilidad ante las distintas fuerzas que debe enfrentar y se constituye en la principal fuente de superioridad a nivel de la industria y la competencia.

Al ser la empresa una organización especial, que emerge de un proceso relacional, el lenguaje del discurso gerencial como herramienta universal para la práctica de la dirección, debe tener en las interacciones cotidianas su fundamento Vidal (2006, p. 138). Para ello, debe desde las diferentes unidades y posiciones, articular con el apoyo de las distintas tecnologías, manejar esos relacionamientos para la acción participativa, que unido a la buena voluntad de todos sus integrantes (Sisto, 2001, p.168) se traduzca en el espacio fértil, para la convivencia, compartir conocimientos, experiencia, aprendizaje, pero sobre todo generar confianza, amistad, tener un buen clima laboral, de abundantes frutos productivos, mantenerse y perdurar.

En este proceso de la empresa, el hombre es el protagonista, las ha creado de acuerdo con su capacidad (Manucci, 2016, p.17), por eso la afirmación de que son creaciones humanas, susceptibles de errores, pueden desaparecer, tienen una vida muy corta, la conceptualiza como empresa productiva, donde el papel del hombre es ser productivo, asimismo, como empresa inteligente y donde el papel del hombre es ser competitivo, por eso también la denominación de agente del cambio. Además, tiene la característica que por ser la empresa un tipo de organización 
especial es la única que se crea para explotar oportunidades de cambio, tanto en la economía como en la sociedad (Drucker y Nakauchi, 1997, p.157), de allí la consideración de contingencia por su alto riesgo y corto periodo de vida, frente a cualquier otro tipo de organización.

\section{METODOLOGÍA}

Es un trabajo que se caracteriza por el análisis de un fenómeno social y colectivo como es la empresa y el aprendizaje, cuyo nudo de relaciones es cualitativo; por lo tanto, la diversidad conceptual que presenta, los hallazgos encontrados, plantean el marco teórico para su análisis, circunscrito dentro del método de la revision documental, el cual, se basó en la recolección de información de tipo secundario, integrada por una bibliografía interdisciplinaria de textos relacionados con la temática y las bases de datos, lo que le da una validez y confiabilidad al documento

\section{RESULTADOS}

La empresa tiene como antecedentes en su surgimiento el desarrollo capitalista, unido al momento histórico la Revolución Industrial, que con la aparición de la máquina a vapor, inicia la teoría de la empresa, y un impulso decisivo a la industrialización. A partir de allí, la empresa empieza a incorporar tecnologías en sus procesos, avanzar en formas más complejas, ser fuente para la explotación de los recursos, y jugar un papel muy importante en la economía de las naciones que hacen de su existencia el sustento y necesidad para contribuir con su prosperidad al progreso y cambio en cada uno de los aspectos de la vida de los ciudadanos.

La empresa es el elemento clave de la sociedad y de las naciones, constituye ese lugar de espacio físico colectivo dinámico, para el encuentro cotidiano, generar gran movimiento de espíritu, para la acción y el aprendizaje, allí las personas trabajan, tienen la esperanza de cercanía, de interacción, aplicar, adquirir conocimiento, compartir experiencias, relacionarse, crear oportunidades, obtener recursos, diseñen procesos abiertos para producir variedad de cosas, satisfacer sus necesidades y el resultado de esa interacción potenciar la capacidad de las personas para proveer a la comunidad bienes y servicios.

\section{DISCUSIÓN}

Dice un viejo proverbio que el más largo de los caminos contempla un paso: la discusión, de considerar la empresa un espacio de aprendizaje. Para ello, la empresa es una comunidad humana que se construye operacionalmente de distintas relaciones e interrelaciones; aunque parezca contradictorio, esta realidad ha sido constatada, en fecha reciente donde se nota un gran interés. El tener la empresa sus antecedentes en el desarrollo capitalista y posteriormente la constitución de un sistema mercantil, le confieren lugar privilegiado en la sociedad de colocar al hombre como administrador para influenciar la vida de las personas, los países, y ser reverenciada la fuente productora de riqueza materiales

La discusión como espacio de aprendizaje aporta que en la convivencia diaria las personas en calidad de ciudadano, colaborador, trabajador, empresario, inversionista, mentor, directivo, operario y cliente, hacen posible el surgimiento de la empresa y las personas captan de ese espacio lo que necesitan para su subsistencia; es decir, el quehacer de las personas en la empresa siempre tiene como referente el espacio, como el gran vehículo para coordinar y mantener una población siempre en aumento en calidad de consumidores, colaboradores, directivos, empresarios y proporcionar bienes y servicios que hacen posibles una vida mejor.

Por otro lado, los diversos y heterogéneos estudios realizados sobre la teoría de la empresa, observado a la luz de su potencial desplegado, la mayoría de ellos fundamentan su desarrollo en la teoría de acción cuantitativa, cuya operación vital da lugar a la producción de bienes y/o servicios, por ello, el énfasis en la eficiencia. Y pone de presente que los estudios sobre la empresa, su base ha sido la gran empresa industrial, bajo la corriente de pensamiento positivista. No obstante, y en respuesta a las condiciones de cambio en el entorno, han surgido nuevos enfoques a partir de la teoría evolutiva y nuevas corrientes de pensamientos que han influenciados la introducción de métodos cualitativos, nuevas visiones y configuraciones alternativas para llevar a cabo las tareas: a nivel micro, pequeña empresa, organizaciones en red, gestión de conocimientos, todos estos argumentos constituyen espacios para la actividad laboral, la inno- 
vación, la investigación, y contribuir hacer de la empresa esa gran fuente potencial de convivencia y aprendizaje

\section{CONCLUSIÓN}

En la actualidad, este trabajo sobre las empresas, como un tipo particular de organización inmersa en un contexto globalizado, tiene la gratitud de discernir algunos signos de los tiempos y mostrar caminos, como lo señala nuestro ser supremo, a empresarios, directivos, colaboradores, investigadores y académicos entre otros. La empresa es considerada un elemento clave para la prosperidad de las personas, la sociedad y las naciones, pero también para la agresividad y dominio sobre la naturaleza por ello, pensamos es un trabajo que debe agradar para la comprensión y mejora tanto de las empresas, como del mundo académico.

Inmersa en un contexto dinámico, la empresa también constituye un sistema integral dinámico no lineal, clave de nuestro tiempo, con cierto grado de rigidez, pero también de flexibilidad, ya que deben enfrentarse en su actuación a unos competidores con características técnicas, organizativas y sociales diferentes. Para su actuación, el elemento fundamental siempre ha sido la persona, que a través de la renovación de su conocimiento y a partir de la Revolución Industrial la ha convertido en una empresa agresiva, especialmente sobre los ecosistemas, en la explotación de materias primas y consumo abusivo de energía.

Desde la perspectiva académica e investigativa se busca llamar la atención, ya que son pocas los esfuerzos dedicados a su conceptualización para una mejor comprensión. Hoy es teorizada como empresa inteligente, para una sociedad del conocimiento, con una concepción del hombre competitivo donde la innovación tiene más protagonismo que la producción en masa; no obstante, en su actuación se aplica la concepción de la empresa industrial moderna que abogó por un sistema productivo con un hombre productivo y calculador. Por eso abrigamos la esperanza de que las facultades de Ciencias Económicas y Administrativas, así como las profesiones afines le dediquen esfuerzos para mejorarlas, es urgente la necesidad de encontrar a través de la empresa caminos de esperanza que hagan sostenible la vida.

\section{REFERENCIAS BIBLIOGRÁFICAS}

Alcaide, J. (2015). Fidelización de clientes. Madrid, España: Editorial ESIC.

Alchian, A. (1965). The basis of some Recent Advances in the Theory of Management of the Firm. Journal of Industrial Economics, (14), 30-41.

Banchik, C., Rainero, M. V., y Escudero, S. (2007). Responsabilidad social empresaria. En Juan S, LLoret., y M. C. Garros (Comp.), Perspectiva sobre derecho ambiental y de la sustentabilidad (pp. 201- 224). Argentina: Ediciones Universidad Católica de Salta.

Bertoglio, J. (2004). Anatomía de la empresa. Una teoría general de las organizaciones. México: Editorial Limusa S.A

Chandler, A. (1987). La mano visible: la revolución de la dirección en la empresa norteamericana. Madrid, España: Ministerio de trabajo y seguridad social.

Chandler, A. D. (1996). Primera parte. Introducción: escala y diversificación. La dinámica del capitalismo industrial. Zaragoza, España: Prensa universitaria de Zaragoza, España.

Claude, S. G. (2005). Historia del pensamiento administrativo. México, D.F: Editorial Pearson/educación.

Coase, R. H. (1937). La naturaleza de la empresa. En 0. E. Williamson., y Sidney, G. Winter (Comp.). La naturaleza de la empresa. Origen evolución y desarrollo (pp. 29-48). México: Fondo de cultura económica.

Commons, J. R. (1931). Institutional economics. American Economic Review, 21, 648-657.

De Geus, A. (1999). La empresa viviente. Buenos Aires, Argentina: Editorial Granica S.A.

Demsetz, H. (1967). Toward a Theory of Property Rights. American Economic Review, (57), 347-358.

Douna, S. y Schreuder, H. (2009). Enfoques económicos para el análisis de las organizaciones. Madrid, España: Editorial Pearson /Prentice-Hall

Drucker, P. F. (1978). La gerencia: tareas, responsabilidades, prácticas. Buenos Aires, Argentina: Editorial El Ateneo.

Drucker, P. y Nakauchi, I. (1997). Drucker habla sobre Asia: dialogo entre Peter Drucker e Isao Nakauchi. Bogotá Colombia: Editorial Norma, S. A.

Emerson, H. (1913). The Twelve principles of efficiency. New York: Engineering Magazine.

Etkin, J. (2011). Gestión de la complejidad en las organizaciones. Buenos Aires Argentina: Editorial Granica.

Fayol, H. (1930). Administración industrial y general. Barcelona, España: Editorial Orbis. 
Friedman, M. y Friedman, D. R. (1998). Libertad de elegir. Bogotá, Colombia: Ediciones Grijalbo, S.A.

Fruin, W. M. (2000). Las fábricas del conocimiento: la administración del capital intelectual. Editorial Oxford. México D, F

García, G. A. y Taboada, E. (2012). Teoría de la empresa: las propuestas de Coase, Alchian y Demsetz, Williamson, Penrose y Nooteboom. Revista Economía: Teoría y Práctica, 36, pp. 9-42. Recuperado de http:// www.redalyc.org/articulo.oa?id=281125196001

García, J. (1994). Historia económica de la empresa moderna. Madrid, España: Ediciones Istmo, S. A.

González, J. (2010). La empresa, marco conceptual y técnica de gestión por áreas funcionales. Barcelona, España: Universidad Autónoma de Barcelona España.

Hall, P. A. (1986). Governing the Economy: The Politics of State Intervention in Britain and France. New York: Oxford University Press.

Jover, D. (2006). Praxis de la esperanza, educación empleo y economía social. Barcelona España: Editorial Icaria, S.A

Juan Pablo II. (1991). Carta encíclica Centesimus Annus. A sus hermanos en el episcopado, al clero, a las familias religiosas. En el centenario de la Rerum Novarum. Recuperado w2.vatican.va/content/jojn-paul-ii/es/ encyclicals/documents/hf_jp-ii_enc_01051991_centesimus-annus.html. Consultado el 03/10/2017

Katz, D. y Kahn, R. (2008). Psicología social de las organizaciones. México, D. F: Editorial Trillas.

Kodman, F. (2012). La empresa consciente: como construir valor a través de valores. Buenos Aires Argentina. Editorial Aguilar

Laval, CH. y Dardot, P. (2013). La nueva razón del mundo. Barcelona, España: Editorial Gedisa.

Martínez, M. (2005). Dirigir empresas de la teoría a la realidad. Madrid, España: Ediciones Internacionales Universitaria S. A.

Manucci, M. (2016). Competitividad emocional. Ediciones B Argentina S.A

Mayo, A. y Lank, E. (2003). Las organizaciones que aprenden. Barcelona, España: Gestión 2000, S.A..

Melé, D. (1991). Orientaciones para dirección de empresas en la encíclica Centesimus Annus. Documento de investigación IESE (223), Universidad de Navarra. Pamplona, España. Recuperado de http://www.iese. edu/research/pdfs/di-0223.pdf

Natoli, S. (2004). Estar en el mundo un paseo por el tiempo presente. Barcelona, España: Editorial Grao de IrIf, S.L
Negri, A. (2006). Fabricas del sujeto: ontología de la subversión. Madrid, España: Ediciones Akal, S.A.

Nonaka, I., y Takeuchi, H. (1995). La empresa creadora de conocimiento. Oxford: University Press

North, D. C. (1994) El desempeño económico a lo largo del tiempo. The American Economic Review, 84(3), 567-583

Ortueta, L. (1972). Organización científica de las empresas. México: Editorial Limusa, Willey, S.A

Pantoja, J., y Arévalo, E. (2006). La empresa moderna en el marco de la corriente institucionalista. EIA, (5). 71-84

Pollard, S. (1987). La génesis de la dirección de empresas moderna. Madrid, España: Ministerio de trabajo y seguridad Social.

Salas, V. (2007). El siglo de las empresas. Bilbao, España: Fundación BBVA.

Sánchez, E. (1990). Joven Empresario Es. Caracas, Venezuela: Producciones, servicios y ediciones económicas.

Sánchez, C. (2002). Deontología, función social y responsabilidad de los profesionales en la empresa. Madrid, España: Universidad complutense de Madrid, España.

Sisto, V. (2001). Teorías organizacionales postmodernas y la gestación del sujeto postmoderno. (Tesis doctoral). Universidad de Barcelona España. Departamento de psicología social. Barcelona, España.

Stewart, A. T. (1997). La nueva riqueza de las organizaciones. El capital intelectual. Ediciones Gránica, S.A. Buenos Aires Argentina.

Tockman, V. (Ed.). (2006). Universalismo básico. Una nueva política social para América Latina. Washington, $D C$ : Editorial Planeta.

Ulrich, D., y Ulrich, W. (2010). El sentido de trabajar. Madrid, España: Editorial Empresarial Lid

Veblen, T. (Ed). (1965). The Portable Veblen. New York: The Viking Press.

Vidal, A. (2006). Prospectivas académicas en la sociedad del conocimiento. Universidad Colegio mayor de Cundinamarca, Bogotá. Recuperado de: www.unicolmayor.edu.co/recursos_user/portal/rec/arc_752.pdf

Williamson, 0. (1991). Mercados y jerarquías: su análisis y sus implicaciones antitrust. México: Fondo de cultura económica.

Wren, D., y Greenwood, R. (1999). Los innovadores de las grandes organizaciones. México. D.F: Editorial Oxford.

Zuluaga, C. (2009). La mano visible: un clásico por debatir en la historiografía empresarial colombiana. Revista de Economía Institucional, 11, (20), 453-459. 